\title{
Influence of receptor selectivity on benefits from SGLT2 inhibitors in patients with heart failure: a systematic review and head-to-head comparative efficacy network meta-analysis
}

\author{
Tobias Täger $^{1} \cdot$ Lutz Frankenstein $^{1}$ (1) $\cdot$ Dan Atar $^{2} \cdot$ Stefan Agewall ${ }^{2} \cdot$ Norbert Frey $^{1} \cdot$ Morten Grundtvig $^{3}$. \\ Andrew L. Clark ${ }^{4}$ John G. F. Cleland ${ }^{5,6} \cdot$ Hanna Fröhlich ${ }^{1}$
}

Received: 28 April 2021 / Accepted: 19 July 2021 / Published online: 8 September 2021

(c) The Author(s) 2021

\begin{abstract}
Background Receptor selectivity of sodium-glucose cotransporter-2 inhibitors (SGLT2i) varies greatly between agents. The overall improvement of cardiovascular (CV) outcomes in heart failure (HF) patients varies between trials. We, therefore, evaluated the comparative efficacy of individual SGLT2i and the influence of their respective receptor selectivity thereon. Methods We identified randomized controlled trials investigating the use of SGLT2i in patients with HF- either as the target cohort or as a subgroup of it. Comparators included placebo or any other active treatment. The primary endpoint was the composite of hospitalization for HF or CV death. Secondary outcomes included all-cause mortality, CV mortality, hospitalization for HF, worsening renal function (RF), and the composite of worsening RF or CV death. Evidence was synthesized using network meta-analysis. In addition, the impact of receptor selectivity on outcomes was analysed using meta-regression. Results We identified 18,265 patients included in 22 trials. Compared to placebo, selective and non-selective SGLT2i improved fatal and non-fatal HF events. Head-to-head comparisons suggest superior efficacy with sotagliflozin as compared to dapagliflozin, empagliflozin or ertugliflozin. No significant difference was found between canagliflozin and sotagliflozin. Meta-regression analyses show a decreasing benefit on HF events with increasing receptor selectivity of SGLT2i. In contrast, receptor selectivity did not affect mortality and renal endpoints and no significant difference between individual SGLT2i was noted.

Conclusion Our data point towards a class-effect of SGLT2i on mortality and renal outcomes. However, non-selective SGLT2i such as sotagliflozin may be superior to highly selective SGLT2i in terms of HF outcomes.
\end{abstract}

Keywords Heart failure $\cdot$ Diabetes mellitus $\cdot$ Sodium glucose cotransporter 2 inhibitor $\cdot$ Receptor selectivity $\cdot$ Outcome

$\begin{array}{ll}\text { Abbreviations } \\ \text { CV } & \text { Cardiovascular } \\ \text { HF } & \text { Heart failure } \\ \text { HFpEF } & \text { Heart failure with preserved ejection fraction } \\ \text { HFrEF } & \text { Heart failure with reduced ejection fraction } \\ \text { LVEF } & \text { Left ventricular ejection fraction }\end{array}$

Tobias Täger and Lutz Frankenstein shared first authorship.

Lutz Frankenstein

lutz.frankenstein@med.uni-heidelberg.de

1 Department of Cardiology, Angiology, and Pneumology, University Hospital Heidelberg, Im Neuenheimer Feld 410, 69120 Heidelberg, Germany

2 Department of Cardiology, Oslo University Hospital Ulleval and Institute of Clinical Sciences, University of Oslo, Oslo, Norway
NMA Network meta-analysis

RCT Randomized controlled trial

SGLT2i Sodium-glucose cotransporter 2 inhibitor

SUCRA Surface under the cumulative ranking probabilities

T2D Type 2 diabetes mellitus 


\section{Introduction}

Sodium-glucose cotransporter-2 inhibitors (SGLT2i) had been established in type 2 diabetes (T2D) care for some time when their positive impact on cardiovascular (CV) outcomes-including heart failure (HF) — was recognized [1-4]. A potential superiority of SGLT2i over other hypoglycemic agents was suspected from observational databases [5] and in the following years, their prognostic value was extended to patients with HF with or without T2D for dapagliflozin in DAPA-HF [6], empagliflozin in EMPEROR-Reduced [7], and sotagliflozin in SOLOISTWHF [8].

Receptor selectivity of SGLT2i varies greatly-while dapagliflozin and empagliflozin are selective SGLT2i, sotagliflozin is a non-selective inhibitor. Moreover, effects on individual CV endpoints differ between trials. It is thus unclear whether (a) the observed benefits represent a drugspecific rather than a class effect and (b) if the extent of the respective $\mathrm{CV}$-benefit is modulated by the individual receptor selectivity. As there are no published prospective head-to-head comparisons of SGLT2i-and to the best of our knowledge none are planned, either-the comparative efficacy of individual SGLT2i in patients with HF remains to be elucidated.

To this end, the direct comparisons of the respective numeric values of effect-measures from the aforementioned trials (so-called naïve comparisons) are statistically inadequate. Standard (conventional) meta-analysis cannot address this question either because the baseline assumption of conventional meta-analysis is a class-effect as it essentially groups verum vs. placebo to calculate a summary effect measure. We, therefore, compared the CV benefits of selective and non-selective SGLT2i in patients with HF using a network meta-analysis (NMA).

\section{Methods}

NMA is an extension of pairwise (conventional) metaanalysis in which multiple interventions are compared both directly within randomized controlled trials (RCTs) and indirectly, across trials, based on a common comparator. NMA has advantages over pair-wise meta-analysis, such as clarification of outcomes from multiple trials including several common comparators and indirect calculation of effects when direct comparisons between important treatments are not available. Also, NMA can provide increased statistical power and cross-validation of the observed treatment-effect of weak connections, given reasonable network connectivity and sufficient sample-sizes. This results in greater precision of treatment-effect estimates and the ability to rank all the interventions in a coherent way.

We performed the present review following the Preferred Reporting Items for Systematic Reviews and Meta-analyses (PRISMA) extension statement for reporting systematic reviews incorporating NMAs of health care interventions [9-12]. The protocol of the NMA was prospectively registered at PROSPERO (registration ID: CRD42020178502).

\section{Identification and selection of trials}

We searched PubMed and www.clinicaltrials.gov up to November 24th 2020 for RCTs investigating the use of gliflozins in patients with HF. Both selective and non-selective SGLT2i were considered. Details of the search strategy are provided in the supplementary material. Two reviewers independently screened citations against the following predefined selection criteria.

Study design We included prospective RCTs investigating treatment with SGLT2i. There were no restrictions regarding the date of publication, language or sample size.

Population We selected trials including adults ( $\geq 18$ years) with a history of HF or a diagnosis of prevalent, stable HF. Patients with HF with reduced, mid-range or preserved left ventricular ejection fraction (LVEF) were considered. There were no restrictions regarding sex, ethnic group, or dose of SGLT2i. Patients with and without diabetes were included. Trials that included solely patients with HF as well subgroups of patients with HF from RCTs with broader inclusion criteria were considered.

Interventions Treatment with either canagliflozin, dapagliflozin, empagliflozin, ertugliflozin, ipragliflozin, luseogliflozin, licogliflozin, remogliflozin, sergliflozin, sotagliflozin or tofogliflozin for at least 12 weeks. An arbitrary limit of 12 weeks was chosen to allow sufficient time for the RCT to accrue events.

Comparators: placebo or standard medical care.

Outcomes: the primary outcome was the composite of hospitalization for HF or CV death. The primary outcome was chosen since it has been reported in large SGLT2i trials and it is relevant to patients with HF. Secondary endpoints included all-cause mortality, CV mortality, hospitalization for HF, worsening renal function (RF), and the composite of worsening RF or CV death. Following the criteria used in trials, worsening RF was defined as doubling of serum creatinine, sustained $40-50 \%$ reduction in the estimated glomerular filtration rate (eGFR), end-stage renal disease, initiation of renal replacement therapy, or renal death.

\section{Data extraction and quality assessment}

All relevant articles were independently reviewed by two investigators to assess the eligibility of the article and 
abstract with standardized data abstraction forms, and disagreement was resolved by a third investigator. For each trial included, details were extracted on trial design, patient characteristics, interventions, and outcomes. The quality of included trials was assessed using the Cochrane Collaboration Criteria [13].

\section{Statistical analyses}

This NMA was conducted with Stata software 15.0 (StataCorp, College Station, TX, USA) using the network family of commands $[14,15]$. A random effects model was applied. The NMA was performed to obtain estimates for outcomes of primary and secondary end-points, presented as odds ratio (OR) and $95 \%$ confidence intervals (CI) for binary outcomes. The plot of a network of drugs was used as a visual representation of the evidence-base and offered a concise description of its characteristics. It consists of nodes representing the interventions being compared and edges representing the available direct comparisons (comparisons evaluated in at least one trial) between pairs of interventions [15-17]. The quality of treatment effect estimates was rated following the Grading of Recommendations Assessment, Development and Evaluation (GRADE) approach $[18,19]$. To make the rank of treatments, we used the surface under the cumulative ranking probabilities (SUCRA) - a transformation of the mean rank that accounts both for the location and the variance of all relative treatment-effects [20]. SUCRA values range from 0 to 1.0. The higher the SUCRA value, and the closer to 1.0, the higher the likelihood that a therapy is in the top rank or one of the top ranks; the closer to 0 the SUCRA value, the more likely that a therapy is in the bottom rank, or one of the bottom ranks [21]. To check for a publication bias, we designed a funnel plot [15]. Consistency of results was evaluated in each loop by calculation of an inconsistency factor and statistical significance determined via $z$ test $[17$, 22].

We evaluated the influence of receptor selectivity on study outcome through meta-regression using the Stata metareg command on study-level summary data [23]. Metaregression determines the extent to which statistical heterogeneity between the values of the respective effect measures of multiple studies can be related to one or more characteristics of the studies-receptor selectivity in our case. To test the stability of the results, we performed sensitivity analyses by restricting analyses to patients with a diagnosis of both $\mathrm{HF}$ and T2D. Data on different dosages of active treatments and/or comparators were pooled for each trial. Inter-rater agreement statistic (Kappa, 95\% CI) was calculated according to Cohen [24]. All $p$ values were two-tailed with the statistical significance arbitrarily set at $<0.05$.

\section{Results}

\section{Literature search}

The search strategy yielded 24 eligible records reporting results from 22 trials [6-8, 25-45]. For two trials, results were not published in a journal article but they were extracted from www.clinicaltrials.gov (NCT03448406 and NCT 03485222). The flowchart of the trial selection process is shown in Online Fig. 1. Agreement between reviewers was excellent ( $\kappa=0.833$, 95\% CI $0.677-0.990)$.

Ten trials investigated the use of SGLT2i in T2D and reported subgroup analyses from a total of 7084 patients with concomitant HF [25-29, 33, 34, 41]. Twelve trials including 11,181 patients prospectively selected patients with HF [6-8, 30-32, 35, 36, 38, 42-45], adding up to a total of 18,265 patients with HF included in the present NMA. All but one trial compared SGLT2i with placebo, and no trials directly compared two different SGLT2i.

Nine trials studied the use of dapagliflozin in a total of 7370 patients with HF. Three trials studied canagliflozin ( $n=2149)$, eight trials studied empagliflozin $(n=5566)$ and one trial studied ertugliflozin in patients with $\operatorname{HF}(n=1958)$. The non-selective SGLT2i licogliflozin and sotagliflozin were each studied in one trial $(n=124$ and $n=1222$, respectively). No trials were identified studying the use of ipragliflozin, luseogliflozin, remogliflozin, sergliflozin, or tofogliflozin in patients with HF. Pharmacokinetic characteristics of SGLT2i included in the present NMA are shown in Online Table 1. For characteristics of trials, please refer to Table 1.

The network plots with respect to different endpoints are presented in Online Fig. 2.

\section{Patient characteristics}

Patients were generally aged between 56 and 74 years and $22-44 \%$ were women. Mean LVEF was reported in nine trials and varied between 26\% [38] and 45\% [43]. Most patients had eGFR $>60 \mathrm{~mL} / \mathrm{min} / 1.73 \mathrm{~m}^{2}$. More than $80 \%$ received treatment with angiotensin-converting enzyme inhibitors, angiotensin receptor blockers, or angiotensin receptor neprilysin inhibitors, and 70-97\% were treated with beta-blockers. The proportion of patients with concomitant T2D was reported in all but two trials $[44,45]$ and varied between 0 and $100 \%$, totaling 12,762 (66.6\%) patients (Online Table 2).

\section{Risk of bias}

The overall risk of bias was low to intermediate. Online Fig. 3 presents the individual items of the risk of bias 





assessment for each trial. Although all data were from RCTs, for ten trials it derived from subgroup analyses [25-29, 33, $34,40,41]$, which, by definition, is not truly randomized. However, eight trials reported baseline characteristics of HF subgroups with respect to trial treatment, all of which demonstrated a good balance of patient characteristics between treatment groups [25-27, 29, 34, 41].

The primary combined endpoint of hospitalization for $\mathrm{HF}$ or $\mathrm{CV}$ death was reported in nine trials including 16,034 patients. All-cause mortality could be retrieved for all but two trials ( $n=15,655$ patients), whereas CV mortality was reported in 13 trials ( $n=14,584$ patients). Data on hospitalization for HF were available for 16,757 patients included in 17 trials, and renal outcomes were reported in five trials ( $n=10,897$ patients). The composite endpoint of worsening $\mathrm{RF}$ or CV death was reported in three $(n=1391$ patients) trials. There was no systematic association between type or size of the trial or the publication date and any pattern of missing endpoint information. The comparison-adjusted funnel plot for the primary endpoint was symmetrical, suggesting the absence of small-trial effects and publication bias (Online Fig. 4).

\section{Outcomes}

\section{Primary outcome}

As shown in Fig. 1, there was a consistent benefit on the composite of hospitalization for HF or CV death across different SGLT2i trials.

Network comparisons of individual SGLT2i included studies on the use of canagliflozin $(n=2 ; 1497$ patients), dapagliflozin ( $n=2 ; 6731$ patients), empagliflozin ( $n=3$; 4626 patients), ertugliflozin ( $n=1 ; 1958$ patients) or sotagliflozin ( $n=1 ; 1222$ patients). No data were available for licogliflozin. The predictive interval plot summarizing the relative mean effects along with the impact of heterogeneity on the respective confidence interval ( $=$ the predictive interval) of each (network) comparison is shown in Fig. 2. When compared to placebo, canagliflozin, dapagliflozin, empagliflozin, and sotagliflozin reduced the composite endpoint of hospitalizations for HF or CV death significantly. A non-significant benefit was found with ertugliflozin. Indirect head-to-head comparisons showed a significant benefit of sotagliflozin over dapagliflozin, empagliflozin, and ertugliflozin. In addition, our results visually suggest a benefit of canagliflozin over dapagliflozin, empagliflozin, and ertugliflozin. However, confidence intervals just cross the line of null effect. No significant difference was found between canagliflozin and sotagliflozin. SUCRA values are presented in Table 2. The graphical display of the ranking based on the SUCRA values is shown in Fig. 3. No closed loops were formed and consequently, no inconsistency could be derived.

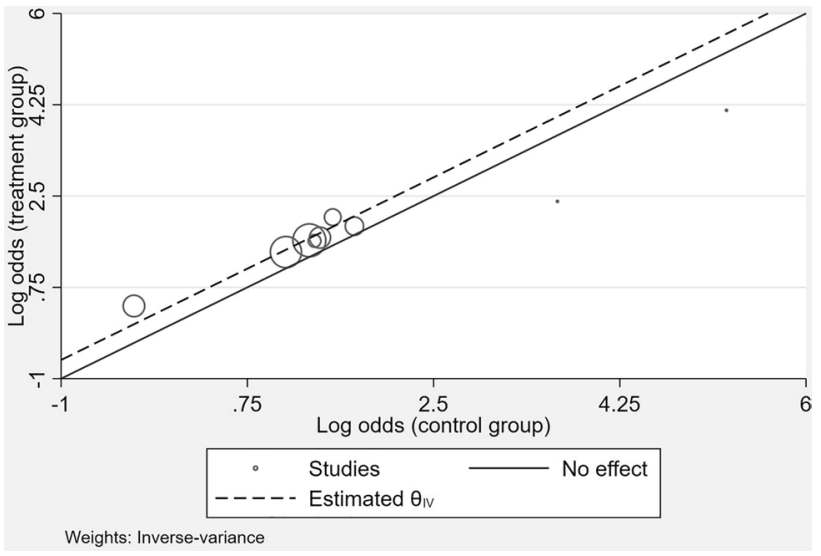

Fig. 1 L'Abbé-plot of trials investigating the effects of SGLT2i on the composite outcome of hospitalizations for $\mathrm{HF}$ or $\mathrm{CV}$ death. $\mathrm{CV}$ cardiovascular, $H F$ heart failure. The l'Abbé-plot plots the event rate in the experimental (intervention) group against the event rate in the control group. Trials in which the experimental treatment proves better than the control will be in the upper left of the plot, between the $y$ axis and the line of equality. If experimental treatment is no better than control then the point will fall on the line of equality, and if control is better than experimental then the point will be in the lower right of the plot, between the $x$ axis and the line of equality. The symbol size represents the sample size of the respective trials

In NMA of selective vs. non-selective SGLT2i, we found that both classes of SGLT2i improve the composite of hospitalization for $\mathrm{HF}$ or $\mathrm{CV}$ death when compared to placebo. However, indirect head-to-head-comparisons show a significant $49 \%$ benefit with non-selective SGLT2i as compared to selective SGLT2i (Fig. 4). Meta-regression analyses revealed a decreasing benefit on the primary outcome with increasing selectivity of SGLT2i (Fig. 5).

\section{Secondary outcomes}

All-cause mortality Comparisons of individual SGLT2i included studies on the use of canagliflozin $(n=2 ; 1497$ patients), dapagliflozin ( $n=9 ; 7370$ patients), empagliflozin ( $n=8 ; 5565$ patients), licogliflozin ( $n=1 ; 124$ patients), and sotagliflozin ( $n=1 ; 1222$ patients). No data were available for ertugliflozin. Although the effect estimates suggest that all SGLT2i improve survival when compared to placebo, the reduction in all-cause mortality was statistically significant only for dapagliflozin (Online Fig. 5). Indirect head-to-head comparisons, however, found no significant differences in survival between agents. The respective SUCRA values are presented in Table 2. The graphical display of the ranking based on the SUCRA values is shown in Online Fig. 6 . Inconsistency could only be derived for one closed loop (empagliflozin-licogliflozin-placebo) and did not reach statistical significance [IF 0.95, 95\% confidence interval (CI) 0.00-4.92]. 
Fig. 2 Predictive interval plot of individual SGLT2i for the combined primary endpoint of hospitalization for $\mathrm{HF}$ or $\mathrm{CV}$ death. $C V$ cardiovascular, $H F$ heart failure, $S G L T 2 i$ sodiumglucose cotransporter 2 inhibitor. The predictive interval plot represents a forest plot of the joint estimated summary effects from both direct and indirect comparisons along with their confidence intervals. Significant summary effects are shown in red

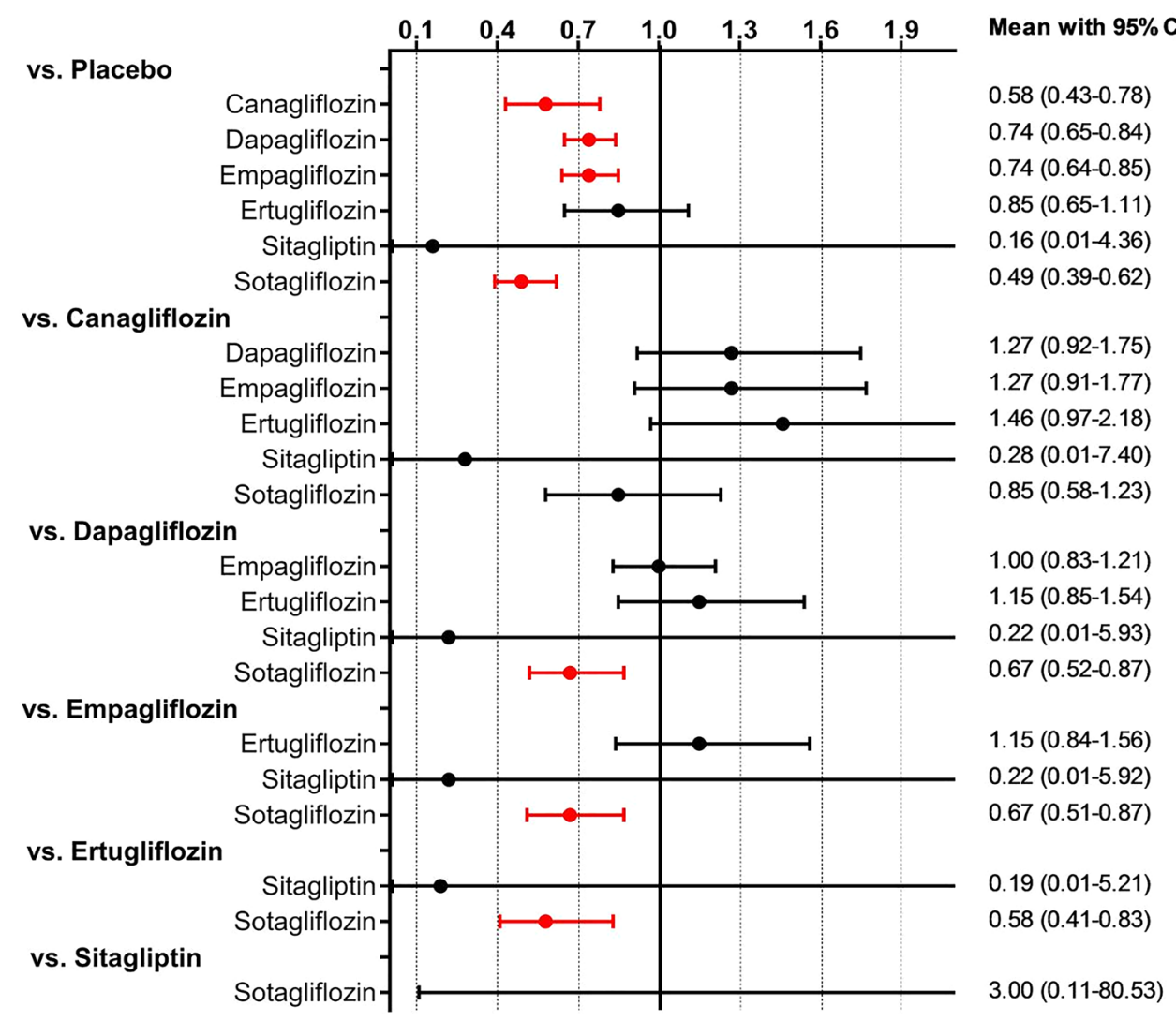

Table 2 Surface under the cumulative ranking curve (SUCRA) values for all endpoints

\begin{tabular}{lllllll}
\hline SUCRA & $\begin{array}{l}\text { Hospitalization } \\
\text { for HF or CV } \\
\text { death }\end{array}$ & $\begin{array}{l}\text { All-cause } \\
\text { mortality }\end{array}$ & CV mortality & $\begin{array}{l}\text { Hospitali- } \\
\text { zation for } \\
\text { HF }\end{array}$ & $\begin{array}{l}\text { Worsening RF } \\
\begin{array}{l}\text { Worsening } \\
\text { RF or CV } \\
\text { death }\end{array}\end{array}$ \\
\hline Canagliflozin & 0.708 & 0.691 & 0.726 & 0.752 & 0.565 & 0.412 \\
Dapagliflozin & 0.432 & 0.611 & 0.608 & 0.337 & 0.442 & n.a. \\
Empagliflozin & 0.425 & 0.386 & 0.503 & 0.347 & 0.672 & 0.439 \\
Ertugliflozin & 0.243 & n.a. & n.a. & 0.473 & n.a. & n.a. \\
Licogliflozin & n.a. & 0.559 & 0.347 & n.a. & n.a. & n.a. \\
Placebo & 0.041 & 0.192 & 0.251 & 0.020 & 0.082 & 0.403 \\
Sitagliptin & 0.809 & 0.558 & 0.550 & 0.819 & 0.739 & 0.671 \\
Sotagliflozin & 0.842 & 0.504 & 0.515 & 0.751 & n.a. & n.a. \\
\hline
\end{tabular}

SUCRA is a transformation of the mean rank that accounts both for the location and the variance of all relative treatment effects. The larger the SUCRA value, the better the rank of the treatment [20]

$C V$ cardiovascular, $H F$ heart failure, n.a. not available, $R F$ renal function
NMA of SGLT2i classes found a $16 \%$ mortality reduction when compared to placebo, with no significant difference between selective and non-selective SGLT2i (Online Fig. 7). Accordingly, meta-regression analyses showed no relationship between effects on mortality and receptor selectivity of SGLT2i (Online Fig. 8).

CV mortality Comparisons of individual SGLT2i included studies on the use of canagliflozin ( $n=2 ; 1497$ patients), dapagliflozin ( $n=4 ; 7050$ patients), empagliflozin ( $n=6$;
5039 patients), licogliflozin ( $n=1 ; 124$ patients), and sotagliflozin $(n=1 ; 1222$ patients). No data were available for ertugliflozin. The results for $\mathrm{CV}$ mortality mirror those for all-cause mortality: When compared to placebo, the reduction in CV mortality was statistically significant only for dapagliflozin. Again, no significant differences in CV mortality were noted between individual SGLT2i (Online Fig. 9). SUCRA values are presented in Table 2. The graphical display of the ranking based on the SUCRA values is shown in Online Fig. 10. Inconsistency could only 


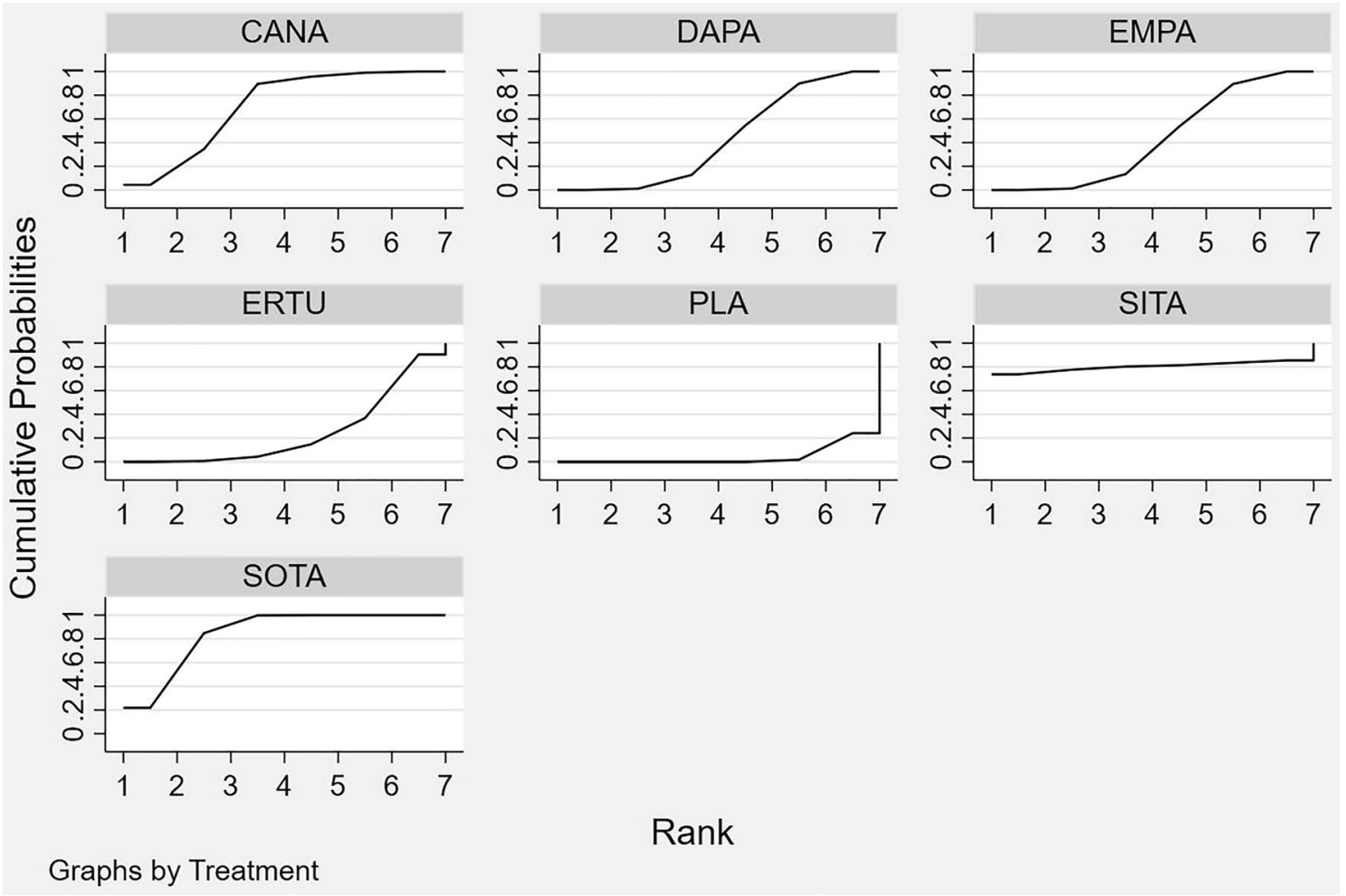

Fig. 3 Graphical ranking of SGLT2i based on SUCRA values (hospitalization for HF or CV death). CANA canagliflozin, CV cardiovascular, $D A P A$ dapagliflozin, EMPA empagliflozin, ERTU ertugliflozin, HF heart failure, PLA placebo, SITA sitagliptin, SOTA sotagliflozin

Fig. 4 Predictive interval plot of selective vs. non-selective SGLT2i for the combined primary endpoint of hospitalization for $\mathrm{HF}$ or $\mathrm{CV}$ death. $C V$ cardiovascular, $H F$ heart failure, $S G L T 2 i$ sodium-glucose cotransporter 2 inhibitor. The predictive interval plot represents a forest plot of the joint estimated summary effects from both direct and indirect comparisons along with their confidence intervals. Significant summary effects are shown in red $\begin{array}{lllllll}0,1 & 0,4 & 0,7 & 1.0 & 1,3 & 1,6 & 1,9\end{array}$

Mean with $95 \% \mathrm{Cl}$

vs. Placebo
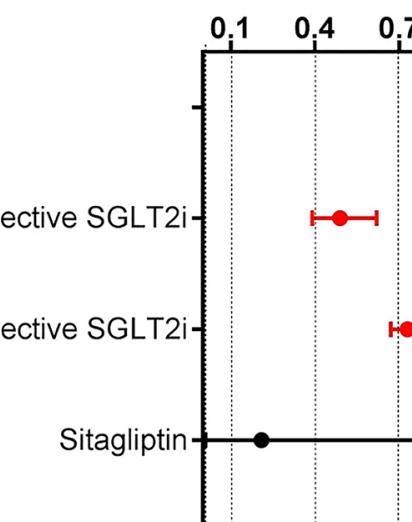

vs. Sitagliptin

non-selective SGLT2i

selective SGLT2i-

vs. non-selective SGLT2i

selective SGLT2i

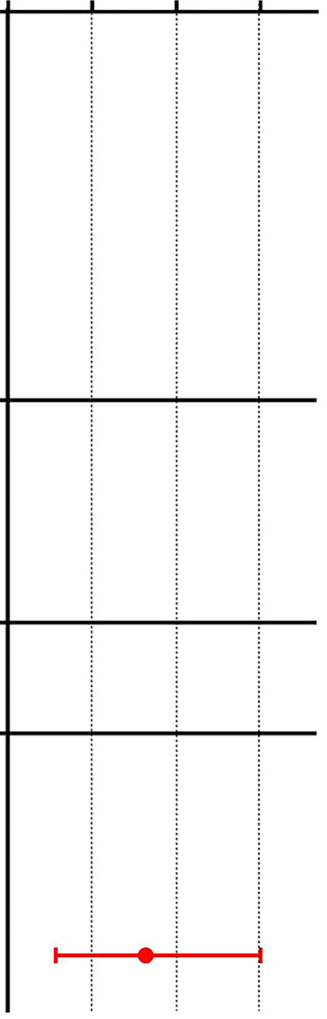

$0.49(0.39-0.62)$

$0.73(0.67-0.80)$

$0.21(0.01-4.53)$

$2.38(0.09-63.00)$

$3.55(0.14-93.01)$

$1.49(1.17-1.90)$ 


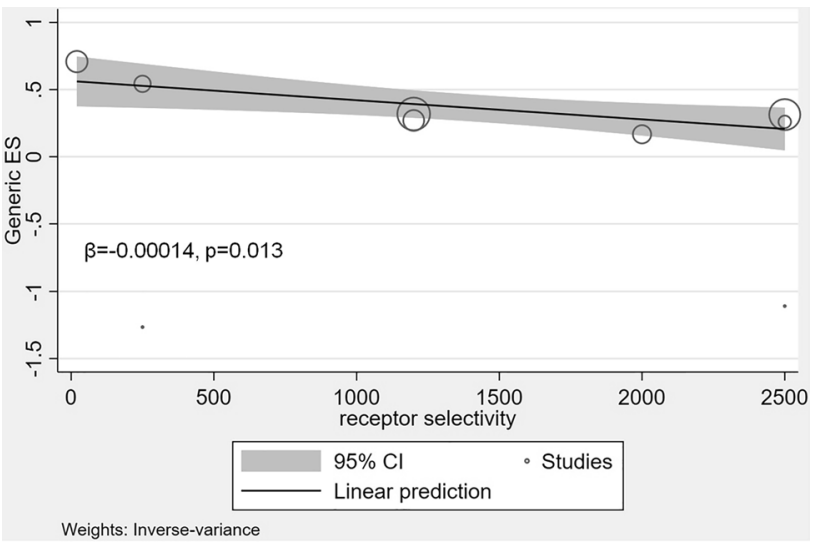

Fig. 5 Relationship between effect size and receptor selectivity of SGLT2i for the combined primary endpoint of hospitalizations for $\mathrm{HF}$ or $\mathrm{CV}$ death. $C I$ confidence interval, $C V$ cardiovascular, $E S$ effect size, $H F$ heart failure, $S G L T 2 i$ sodium-glucose cotransporter 2 inhibitor. Each bubble represents an SGLT2i trial. The symbol size represents the sample size of the respective trials

be derived for one closed loop (empagliflozin-licogliflozin-placebo) and did not reach statistical significance (IF $0.21,95 \%$ CI $0.00-4.78)$. There was no relationship between CV mortality and receptor selectivity of SGLT2i (Online Figs. 11 and 12).

Hospitalization for HF Comparisons of individual SGLT2i included studies on the use of canagliflozin $(n=2 ; 1497$ patients), dapagliflozin ( $n=9 ; 7370$ patients), empagliflozin ( $n=4 ; 4710$ patients), ertugliflozin ( $n=1 ; 1958$ patients) or sotagliflozin ( $n=1 ; 1222$ patients). No data were available for licogliflozin. The results for hospitalization for HF mirror those for the combined outcome of hospitalization for HF or CV death: As shown in Online Fig. 13, all SGLT2i reduced hospitalizations for HF when compared to placebo, with sotagliflozin being superior to dapagliflozin and empagliflozin. The respective SUCRA values are presented in Table 2. The graphical display of the ranking based on the SUCRA values is shown in Online Fig. 14. No closed loops were formed and consequently, no inconsistency could be derived. NMA of selective vs. non-selective SGLT2i shows that both classes of SGLT2i improve hospitalizations for HF when compared to placebo, with a significant $36 \%$ benefit of non-selective SGLT2i over selective SGLT2i (Online Fig. 15). Again, the effect on HF events decreased with increasing receptor selectivity of SGLT2i (Online Fig. 16).

Worsening RF Comparisons of individuals included studies on the use of canagliflozin $(n=2 ; 1497$ patients), dapagliflozin ( $n=2 ; 5007$ patients) or empagliflozin $(n=2 ; 4429$ patients). No data were available for ertugliflozin, licogliflozin or sotagliflozin. As displayed in the predictive interval plot (Online Fig. 17), canagliflozin, dapagliflozin and empa- gliflozin reduced worsening RF when compared to placebo with only empagliflozin reaching statistical significance. Indirect head-to-head comparisons found no significant differences in renal outcomes between individual SGLT2i. SUCRA values are presented in Table 2. The graphical display of the ranking based on the SUCRA values is shown in Online Fig. 18. No closed loops were formed and consequently, no inconsistency could be derived. Due to missing data, NMA of selective vs. non-selective SGLT2i were not possible. Meta-regression analyses showed no significant relationship between receptor selectivity and worsening RF in patients with HF (Online Fig. 19). However, due to the limited number of studies included in the analysis, confidence intervals are wide and results need to be interpreted with caution.

Worsening RF or CV death Only three trials on the use of canagliflozin ( $n=2 ; 688$ patients) or empagliflozin ( $n=1$; 699 patients) in patients with HF reported the composite outcome of worsening RF and CV death $[25,30,40]$. Therefore, NMA calculations using a random effects model were not possible. The following results were obtained using a fixed-effects model and should be interpreted with caution. The predictive interval plot (Online Fig. 20) showed no significant effect of canagliflozin and empagliflozin on the composite endpoint of worsening RF or CV death when compared to placebo. There were no significant differences in the composite outcome between individual SGLT2i. SUCRA values are presented in Table 2. No closed loops were formed and consequently, no inconsistency could be derived. Due to missing data, NMA of selective vs. nonselective SGLT2i, as well as meta-regression analyses, were not possible.

For all endpoints including the respective outcome numbers per trial arm please also refer to Online Table 3.

\section{Sensitivity analyses}

Sensitivity analyses restricting analyses to patients with concomitant HF and T2D essentially confirmed our main results. However, confidence intervals of effect estimates with respect to HF or renal endpoints were wide and results thus need to be interpreted with caution. Detailed results from sensitivity analyses are presented in the supplementary material (supplemental results, Online Figs. 21-30).

\section{Discussion}

Currently, no data exists on either the impact of receptor selectivity on the magnitude of risk reduction or the comparative CV effectiveness of individual SGLT2i-neither in patients with HF nor T2D. Using data from 18,265 patients 
enrolled in 22 trials, we found that all SGLT2i improve fatal and non-fatal HF events when compared to placebo. However, the benefits of SGLT2i increase with decreasing receptor selectivity, with sotagliflozin showing superior efficacy on HF outcomes. In contrast, receptor selectivity does not affect mortality or renal endpoints and no significant difference between individual SGLT2i was noted. It is here that our NMA significantly supplements and extents existing, conventional meta-analyses $[46,47]$ as it includes substantially more trials and patients and it provides statistical evidence for their underlying assumption-a class-effect-via NMA as the most appropriate tool.

To date, the mechanisms behind the benefits of SGLT2i in HF are not fully understood. Considering that SGLT2 receptors are not expressed in the human myocardium [48], it is difficult to reconcile whether and how SGLT2 inhibition could have direct effects on ventricular function [49]. In a randomized study on 56 patients with HF with reduced ejection fraction (HFrEF) and T2D, dapagliflozin did not have a significant effect on left ventricular remodelling as determined by cardiac MRI [43]. In addition, empagliflozin did not change NTproBNP levels in patients with mildly symptomatic HFrEF included in EMPIRE-HF [32]. Some authors have postulated that SGLT2i-mediated cardioprotective effects are secondary to indirect effects on sodium and calcium entry into cardiomyocyte cellular compartments [49]. Others propose that SGLT2i improve the efficiency of myocardial energetics by inducing a favourable shift in glucose and fat metabolism towards increased ketone substrate use [50-52]. Improved endothelial function and reduced vascular stiffening as well as increased diuresis and erythropoietin production may further contribute to the $\mathrm{CV}$ benefits seen with SGLT2i [49]. The combined inhibition of SGLT1 and SGLT2 is hypothesized to enhance the effects on renal sodium and glucose handling further via inhibition of both cotransporter subtypes in the proximal renal tubule $[31,53]$. SGLT1 on the other hand has an important role in glucose absorption in the intestines-and in contrast to SGLT2, SGLT1 receptors are specifically expressed in the human myocardium $[54,55]$. Left ventricular SGLT1 appeared upregulated in patients with HF in 71 patients with end-stage HF [56]. Here, ventricular myocardial SGLT1 expression correlated significantly with measures of cardiac remodelling and systolic function [56]. Whether and how the cardiac expression of SGLT1 contributes to the pronounced benefits seen with sotagliflozin in patients with HF needs to be elucidated. With respect to the dual SGLTi licogliflozin, investigations on the effects on HF events in patients with a prior diagnosis of HF are no longer pursued by the pharmaceutical developer and the company is focussing further research on patients with hepatic steatosis.

While results for HF-endpoints were robust, results for all-cause mortality were largely driven by DAPA-HF, which is the largest trial to date $(n=4744)$ that included solely patients with HFrEF with or without T2D [6]. In DAPA$\mathrm{HF}$, all-cause mortality was reduced by $17 \%$ and $\mathrm{CV}$ death by $18 \%$ with dapagliflozin. In contrast, the EMPERORReduced trial reported a non-significant $8 \%$ reduction in all-cause and CV death with empagliflozin in patients with symptomatic HFrEF [7]. The recently published SOLOISTWHF trial found a $18 \%$ reduction in deaths from any causes and a $16 \%$ reduction in $\mathrm{CV}$ death in patients with $\mathrm{T} 2 \mathrm{D}$ who had recently been hospitalized for worsening HF [8]. However, due to the relatively small number of patients included in the trial $(n=1222)$, confidence intervals were wide and results did not reach statistical significance. To date, there are no prospective $\mathrm{CV}$ outcome trials with canagliflozin, ertugliflozin or licogliflozin in patients with HF. In largescale trials involving patients with $\mathrm{T} 2 \mathrm{D}$, the risk reductions in $\mathrm{CV}$ death among patients with $\mathrm{HF}$ at baseline were $28 \%$ for canagliflozin, $45 \%$ for dapagliflozin, and $29 \%$ for empagliflozin, respectively [27, 33, 41]. In our study, indirect head-to-head comparisons including 15,655 patients from 20 trials revealed no significant differences in mortality reduction between individual SGLT2i. In addition, there was no difference between selective and non-selective SGLT2i with respect to all-cause or CV mortality. The different effects on mortality in DAPA-HF, EMPEROR-Reduced, and SOLOIST-WHF may thus be explained by differences in trial sizes and populations, as indicated by a significantly higher event rate in EMPEROR-Reduced and SOLOIST-WHF as compared to DAPA-HF [6-8].

For the prevention of worsening RF, the mean effect estimates in our analysis point towards a benefit with canagliflozin, dapagliflozin and empagliflozin compared to placebo, with only empagliflozin reaching statistical significance. No data were available for ertugliflozin, licogliflozin or sotagliflozin. Our results are largely driven by the EMPERORReduced trial that observed a lower risk of the composite renal outcome in the empagliflozin group than in the placebo group [7]. In contrast, DAPA-HF reported a significantly greater increase in serum creatinine at eight months in those assigned to dapagliflozin compared to placebo [6]. Then again, the number of adverse renal events was similar between treatment groups [6]. In the CREDENCE trial, canagliflozin improved the composite primary endpoint of worsening RF or CV death in patients with T2D [40]. However, the number of events in the subgroup of patients with T2D and HF were similar between treatment groups (52 events with canagliflozin vs. 53 events with placebo). Again, we found no relationship between selectivity for SGLT2 and renal outcomes. However, the number of trials and patients in analyses was limited and should therefore be interpreted with caution. Further research is needed to clarify the effects of SGLT2i and the role of receptor selectivity on renal function in patients with HF. 
Our study has several potential limitations. First, data for ertugliflozin, licogliflozin and sotagliflozin each stem from only one trial and are therefore susceptible to bias. Sotagliflozin is the only non-selective SGLT2i reporting data on HF outcomes and to the best of our knowledge, no other non-selective SGLT2i are currently being studied in patients with HF. The number of patients included in SOLOIST-WHF was rather small $(n=1222)$ and follow-up was limited to nine months due to loss of funding from the sponsor. In addition, SOLOIST-WHF was the only trial that enrolled patients admitted for acute HF, whereas other trials included patients with chronic stable HF. The selection of acutely decompensated HF patients, as well as the early termination of the trial, may have exaggerated the treatment effects of sotagliflozin. Results should therefore be interpreted with caution until more evidence on the use of sotagliflozin (or other non-selective SGTL2i) in patients with HF is available. Second, we identified twelve trials that solely included patients with HF, whereas ten trials were trials of T2DM that reported results for HF subgroups. By definition, randomization is invalid in subgroup analyses, thereby increasing the risk of bias. However, eight of these trials reported baseline characteristics of HF subgroups with respect to trial treatment, which demonstrated a good balance between treatment groups [25-27, 29, 34, 41]. Most importantly, background HF treatment was similar amongst prospective SGLT2i trials of HF and subgroup analyses of HF in SGLT2i trials of T2DM, with $>80 \%$ of patients receiving renin-angiotensin-aldosterone-system inhibitors and beta-blockers. Third, LVEF was reported in only nine trials, of which all but one enrolled patients with HFrEF. None of the T2D CV outcome trials reported LVEF. It is currently uncertain whether the effects of SGLT2 on HF outcomes differ for patients with reduced versus preserved LVEF (HFpEF). Recently, the trial sponsors Boehringer Ingelheim and Eli Lilly and Company announced that empagliflozin failed to improve exercise capacity as measured by $6 \mathrm{~min}$ walking distance in the EMPERIAL trials. EMPERIAL consisted of two phase III randomized, double-blind trials that included patients with HFrEF (EMPERIAL-reduced) or HFpEF (EMPERIAL-preserved) with our without diabetes. The number of patients included, however, was modest (312 in EMPERIAL-reduced and 315 in EMPERIAL-preserved), and follow-up was only 12 weeks. Then again, a subgroup analysis of the DECLARE-TIMI 58 trial showed that dapagliflozin improved CV outcomes in patients with HFrEF but not in those with HFpEF at baseline [33]. Ongoing trials such as the EMPEROR-preserved trial with empagliflozin and the DELIVER trial with dapagliflozin will help to clarify the role of SGLT2i in HFpEF.

\section{Conclusion}

In conclusion, our data point towards a class-effect of SGLT2i on mortality and renal outcomes. However, nonselective SGLT2i such as sotagliflozin may be superior to highly selective SGLT2i in terms of HF outcomes. More studies are warranted to clarify the role of receptor selectivity of SGLT2i in the treatment of patients with HF.

Supplementary Information The online version contains supplementary material available at https://doi.org/10.1007/s00392-021-01913-z.

Author contributions TT and LF: literature search, data extraction, statistical analyses, drafting of the manuscript. DA, SA, NF, MG, ALC, JGFC: revision of the manuscript for important intellectual content. HF: conception and design, literature search, data extraction, statistical analyses, revision of the manuscript for important intellectual content, supervision.

Funding Open Access funding enabled and organized by Projekt DEAL. None.

Data availability The present study is a network meta-analysis of published randomized trials. All data used for analyses are presented in the manuscript.

Code availability Not available.

\section{Declarations}

Conflict of interest Prof. Atar reports personal fees from Astra-Zeneca, personal fees from Boehringer-Ingelheim, personal fees from Novartis, and personal fees from MSD (outside the submitted work). Prof. Cleland reports personal fees from Abbott, personal fees from Amgen, grants and personal fees from Bayer, grants and personal fees from Bristol Myers Squibb, personal fees from Novartis, personal fees from Medtronic, personal fees from Idorsia, grants and personal fees from Vifor, grants and personal fees from Pharmacosmos, grants and personal fees from Cytokinetics, personal fees from Servier, non-financial support from Boehringer-Ingelheim, personal fees from Astra-Zeneca, personal fees from Innolife, personal fees from Torrent, grants and personal fees from Johnson \& Johnson, grants and personal fees from Myokardia, personal fees from Respicardia, grants and personal fees from Stealth Biopharmaceuticals, and grants and personal fees from Viscardia (outside the submitted work). Prof. Frankenstein reports personal fees from Astra-Zeneca, grants and personal fees from Bayer, personal fees from Boehringer (outside the submitted work). Prof. Frey reports personal fees from Boehringer and personal fees from AstraZeneca (outside the submitted work).

Open Access This article is licensed under a Creative Commons Attribution 4.0 International License, which permits use, sharing, adaptation, distribution and reproduction in any medium or format, as long as you give appropriate credit to the original author(s) and the source, provide a link to the Creative Commons licence, and indicate if changes were made. The images or other third party material in this article are included in the article's Creative Commons licence, unless indicated otherwise in a credit line to the material. If material is not included in the article's Creative Commons licence and your intended use is not permitted by statutory regulation or exceeds the permitted use, you will need to obtain permission directly from the copyright holder. To view a copy of this licence, visit http://creativecommons.org/licenses/by/4.0/. 


\section{References}

1. Zelniker TA, Wiviott SD, Raz I, Im K, Goodrich EL, Bonaca MP et al (2019) SGLT2 inhibitors for primary and secondary prevention of cardiovascular and renal outcomes in type 2 diabetes: a systematic review and meta-analysis of cardiovascular outcome trials. Lancet (London, England) 393(10166):31-39

2. Neal B, Perkovic V, Mahaffey KW, de Zeeuw D, Fulcher G, Erondu $\mathrm{N}$ et al (2017) Canagliflozin and cardiovascular and renal events in type 2 diabetes. N Engl J Med 377(7):644-657

3. Wiviott SD, Raz I, Bonaca MP, Mosenzon O, Kato ET, Cahn A et al (2019) Dapagliflozin and cardiovascular outcomes in type 2 diabetes. N Engl J Med 380(4):347-357

4. Zinman B, Wanner C, Lachin JM, Fitchett D, Bluhmki E, Hantel S et al (2015) Empagliflozin, cardiovascular outcomes, and mortality in type 2 diabetes. N Engl J Med 373(22):2117-2128

5. Ryan PB, Buse JB, Schuemie MJ, DeFalco F, Yuan Z, Stang $P E$ et al (2018) Comparative effectiveness of canagliflozin, SGLT2 inhibitors and non-SGLT2 inhibitors on the risk of hospitalization for heart failure and amputation in patients with type 2 diabetes mellitus: a real-world meta-analysis of 4 observational databases (OBSERVE-4D). Diabetes Obes Metab 20(11):2585-2597

6. McMurray JJV, Solomon SD, Inzucchi SE, Kober L, Kosiborod MN, Martinez FA et al (2019) Dapagliflozin in patients with heart failure and reduced ejection fraction. N Engl J Med 381:1995-2008

7. Packer M, Anker SD, Butler J, Filippatos G, Pocock SJ, Carson P et al (2020) Cardiovascular and renal outcomes with empagliflozin in heart failure. N Engl J Med 383:1413-1424

8. Bhatt DL, Szarek M, Steg PG, Cannon CP, Leiter LA, McGuire DK et al (2020) Sotagliflozin in patients with diabetes and recent worsening heart failure. N Engl J Med 384:117-128

9. Hutton B, Catala-Lopez F, Moher D (2016) The PRISMA statement extension for systematic reviews incorporating network meta-analysis: PRISMA-NMA. Med Clin (Barc) 147(6):262-266

10. Moher D, Stewart L, Shekelle P (2016) Implementing PRISMA-P: recommendations for prospective authors. Syst Rev 5:15

11. Shamseer L, Moher D, Clarke M, Ghersi D, Liberati A, Petticrew $M$ et al (2015) Preferred reporting items for systematic review and meta-analysis protocols (PRISMA-P) 2015: elaboration and explanation. BMJ 349:g7647

12. Moher D, Shamseer L, Clarke M, Ghersi D, Liberati A, Petticrew $M$ et al (2015) Preferred reporting items for systematic review and meta-analysis protocols (PRISMA-P) 2015 statement. Syst Rev $4: 1$

13. Higgins J, Green S (eds) (2011) Cochrane handbook for systematic reviews of interventions version 5.1.0. The Cochrane Collaboration (updated March 2011)

14. Higgins JP, Jackson D, Barrett JK, Lu G, Ades AE, White IR (2012) Consistency and inconsistency in network meta-analysis: concepts and models for multi-arm studies. Res Synth Methods 3(2): $98-110$

15. Chaimani A, Higgins JP, Mavridis D, Spyridonos P, Salanti G (2013) Graphical tools for network meta-analysis in STATA. PLoS ONE 8(10):e76654

16. Salanti G (2012) Indirect and mixed-treatment comparison, network, or multiple-treatments meta-analysis: many names, many benefits, many concerns for the next generation evidence synthesis tool. Res Synth Methods 3(2):80-97

17. Salanti G, Marinho V, Higgins JP (2009) A case study of multipletreatments meta-analysis demonstrates that covariates should be considered. J Clin Epidemiol 62(8):857-864
18. Salanti G, Del Giovane C, Chaimani A, Caldwell DM, Higgins JP (2014) Evaluating the quality of evidence from a network metaanalysis. PLoS ONE 9(7):e99682

19. Puhan MA, Schunemann HJ, Murad MH, Li T, BrignardelloPetersen R, Singh JA et al (2014) A GRADE Working Group approach for rating the quality of treatment effect estimates from network meta-analysis. BMJ 349:g5630

20. Salanti G, Ades AE, Ioannidis JP (2011) Graphical methods and numerical summaries for presenting results from multiple-treatment meta-analysis: an overview and tutorial. J Clin Epidemiol 64(2):163-171

21. Mbuagbaw L, Rochwerg B, Jaeschke R, Heels-Andsell D, Alhazzani W, Thabane L et al (2017) Approaches to interpreting and choosing the best treatments in network meta-analyses. Syst Rev 6(1):79

22. Song F, Harvey I, Lilford R (2008) Adjusted indirect comparison may be less biased than direct comparison for evaluating new pharmaceutical interventions. J Clin Epidemiol 61(5):455-463

23. Harbord RM, Higgins JPT (2008) Meta-regression in Stata. Stata J 8(4):493-519

24. Cohen J (1960) A coefficient of agreement for nominal scales. Educ Psychol Measur 20:37-46

25. Butler J, Zannad F, Fitchett D, Zinman B, Koitka-Weber A, von Eynatten $M$ et al (2019) Empagliflozin improves kidney outcomes in patients with or without heart failure. Circ Heart Fail 12(6): 0005875

26. Fitchett D, Butler J, van de Borne P, Zinman B, Lachin JM, Wanner $C$ et al (2018) Effects of empagliflozin on risk for cardiovascular death and heart failure hospitalization across the spectrum of heart failure risk in the EMPA-REG OUTCOME(R) trial. Eur Heart J 39(5):363-370

27. Fitchett D, Zinman B, Wanner C, Lachin JM, Hantel S, Salsali A et al (2016) Heart failure outcomes with empagliflozin in patients with type 2 diabetes at high cardiovascular risk: results of the EMPA-REG OUTCOME(R) trial. Eur Heart J 37(19):1526-1534

28. Cannon CP, Pratley R, Dagogo-Jack S, Mancuso J, Huyck S, Masiukiewicz U et al (2020) Cardiovascular outcomes with ertugliflozin in type 2 diabetes. N Engl J Med 383(15):1425-1435

29. Cosentino F, Cannon CP, Cherney DZI, Masiukiewicz U, Pratley R, Jack SD et al (2020) Efficacy of ertugliflozin on heart failure-related events in patients with type 2 diabetes mellitus and established atherosclerotic cardiovascular disease: results of the VERTIS CV trial. Circulation 142:2205-2215

30. Carbone S, Billingsley HE, Canada JM, Bressi E, Rotelli B, Kadariya D et al (2020) The effects of canagliflozin compared to sitagliptin on cardiorespiratory fitness in type 2 diabetes mellitus and heart failure with reduced ejection fraction: the CANA-HF study. Diabetes Metab Res Rev 36:e3335

31. de Boer RA, Núñez J, Kozlovski P, Wang Y, Proot P, Keefe D (2020) Effects of the dual sodium-glucose linked transporter inhibitor, licogliflozin vs placebo or empagliflozin in patients with type 2 diabetes and heart failure. Br J Clin Pharmacol 86(7):1346-1356

32. Jensen J, Omar M, Kistorp C, Poulsen MK, Tuxen C, Gustafsson I et al (2020) Twelve weeks of treatment with empagliflozin in patients with heart failure and reduced ejection fraction: a doubleblinded, randomized, and placebo-controlled trial. Am Heart J 228:47-56

33. Kato ET, Silverman MG, Mosenzon O, Zelniker TA, Cahn A, Furtado RHM et al (2019) Effect of dapagliflozin on heart failure and mortality in type 2 diabetes mellitus. Circulation 139(22):2528-2536

34. Kosiborod M, Gause-Nilsson I, Xu J, Sonesson C, Johnsson E (2017) Efficacy and safety of dapagliflozin in patients with type 2 diabetes and concomitant heart failure. J Diabetes Complicat 31(7):1215-1221 
35. Lee MMY, Brooksbank KJM, Wetherall K, Mangion K, Roditi G, Campbell RT et al (2020) Effect of empagliflozin on left ventricular volumes in patients with type 2 diabetes, or prediabetes, and heart failure with reduced ejection fraction (SUGAR-DM-HF). Circulation 143:516-525

36. McMurray JJV, DeMets DL, Inzucchi SE, Kober L, Kosiborod MN, Langkilde AM et al (2019) The dapagliflozin and prevention of adverse-outcomes in heart failure (DAPA-HF) trial: baseline characteristics. Eur J Heart Fail 21:1402-1411

37. Petrie MC, Verma S, Docherty KF, Inzucchi SE, Anand I, Belohlávek J et al (2020) Effect of dapagliflozin on worsening heart failure and cardiovascular death in patients with heart failure with and without diabetes. JAMA 323(14):1353-1368

38. Nassif ME, Windsor SL, Tang F, Khariton Y, Husain M, Inzucchi SE et al (2019) Dapagliflozin effects on biomarkers, symptoms, and functional status in patients with heart failure with reduced ejection fraction: the DEFINE-HF trial. Circulation 140(18):1463-1476

39. Anker SD, Butler J, Filippatos G, Khan MS, Marx N, Lam CSP et al (2020) Effect of empagliflozin on cardiovascular and renal outcomes in patients with heart failure by baseline diabetes status-results from the EMPEROR-reduced trial. Circulation 143:337-349

40. Perkovic V, Jardine MJ, Neal B, Bompoint S, Heerspink HJL, Charytan DM et al (2019) Canagliflozin and renal outcomes in type 2 diabetes and nephropathy. N Engl J Med 380(24):2295-2306

41. Radholm K, Figtree G, Perkovic V, Solomon SD, Mahaffey KW, de Zeeuw D et al (2018) Canagliflozin and heart failure in type 2 diabetes mellitus. Circulation 138(5):458-468

42. Santos-Gallego CG, Vargas-Delgado AP, Requena JA, GarciaRopero A, Mancini D, Pinney S et al (2020) Randomized trial of empagliflozin in non-diabetic patients with heart failure and reduced ejection fraction. J Am Coll Cardiol 77:243-255

43. Singh JSS, Mordi IR, Vickneson K, Fathi A, Donnan PT, Mohan $M$ et al (2020) Dapagliflozin versus placebo on left ventricular remodeling in patients with diabetes and heart failure: the REFORM trial. Diabetes Care 43(6):1356-1359

44. Boehringer Ingelheim. A phase III randomised, double-blind trial to evaluate the effect of 12 weeks treatment of once daily empagliflozin $10 \mathrm{mg}$ compared with placebo on exercise ability and heart failure symptoms, in patients with chronic heart failure with reduced ejection fraction (HFrEF) (EMPERIAL-reduced) 2020. https://clinicaltrials.gov/ct2/show/results/NCT03448419?term $=\%$ 22Empagliflozin $\% 22+\mathrm{OR}+\% 22$ Dapagliflozin $\% 22+\mathrm{OR}+\% 22 \mathrm{Can}$ agliflozin $\% 22+\mathrm{OR}+\%$ 22Ertugliflozin $\% 22+\mathrm{OR}+\%$ 22Ipraglif lozin $\% 22+$ OR+\%22Remogliflozin $\% 22+O R+\% 22$ Sergliflozin $\%$ $22+\mathrm{OR}+\% 22$ Sotagliflozin $\% 22+\mathrm{OR}+\% 22$ Tofogliflozin $\% 22+$ OR+\%22Luseogliflozin $\% 22+\mathrm{OR}+\% 22 \mathrm{Licogliflozin} \% 22 \& \mathrm{recrs}=$ eh\&type $=\operatorname{Intr} \&$ cond $=$ heart + failure $\&$ age $=12 \&$ draw $=2 \&$ rank $=2$. Accessed 1 Dec 2020

45. Boehringer Ingelheim. A phase III randomised, double-blind trial to evaluate the effect of 12 weeks treatment of once daily empagliflozin $10 \mathrm{mg}$ compared with placebo on exercise ability and heart failure symptoms, in patients with chronic heart failure with preserved ejection fraction (HFpEF) (EMPERIAL - Preserved) 2020. https://clinicaltrials.gov/ct2/show/NCT02920918?term=\%22Emp agliflozin $\% 22+\mathrm{OR}+\% 22 \mathrm{Dapagliflozin} \% 22+\mathrm{OR}+\% 22 \mathrm{Canaglif}$ lozin $\% 22+\mathrm{OR}+\%$ 22Ertugliflozin $\% 22+\mathrm{OR}+\% 22$ Ipragliflozin $\%$ $22+\mathrm{OR}+\% 22 \mathrm{Remogliflozin} \% 22+\mathrm{OR}+\% 22$ Sergliflozin $\% 22+$ $\mathrm{OR}+\% 22$ Sotagliflozin $\% 22+\mathrm{OR}+\% 22$ Tofogliflozin $\% 22+\mathrm{OR}+\%$ 22Luseogliflozin\%22+OR+\%22Licogliflozin $\% 22 \&$ recrs=eh\& type $=$ Intr $\&$ cond $=$ heart + failure $\&$ age $=12 \&$ draw $=3 \&$ rank $=17$. Accessed 1 Dec 2020

46. Kumar K, Kheiri B, Simpson TF, Osman M, Rahmouni H (2020) Sodium-glucose cotransporter-2 inhibitors in heart failure: a meta-analysis of randomized clinical trials. Am J Med 133(11):e625-e630

47. Butler J, Usman MS, Khan MS, Greene SJ, Friede T, Vaduganathan $M$ et al (2020) Efficacy and safety of SGLT2 inhibitors in heart failure: systematic review and meta-analysis. ESC Heart Fail 7(6):3298-3309

48. Di Franco A, Cantini G, Tani A, Coppini R, Zecchi-Orlandini S, Raimondi L et al (2017) Sodium-dependent glucose transporters (SGLT) in human ischemic heart: a new potential pharmacological target. Int J Cardiol 243:86-90

49. Lytvyn Y, Bjornstad P, Udell JA, Lovshin JA, Cherney DZI (2017) Sodium glucose cotransporter-2 inhibition in heart failure: potential mechanisms, clinical applications, and summary of clinical trials. Circulation 136(17):1643-1658

50. Packer M, Anker SD, Butler J, Filippatos G, Zannad F (2017) Effects of sodium-glucose cotransporter 2 inhibitors for the treatment of patients with heart failure: proposal of a novel mechanism of action. JAMA Cardiol 2(9):1025-1029

51. Martens P, Mathieu C, Verbrugge FH (2017) Promise of SGLT2 inhibitors in heart failure: diabetes and beyond. Curr Treat Options Cardiovasc Med 19(3):23

52. Packer M (2019) Reconceptualization of the molecular mechanism by which sodium-glucose cotransporter 2 inhibitors reduce the risk of heart failure events. Circulation 140(6):443-445

53. He YL, Haynes W, Meyers CD, Amer A, Zhang Y, Mahling P et al (2019) The effects of licogliflozin, a dual SGLT1/2 inhibitor, on body weight in obese patients with or without diabetes. Diabetes Obes Metab 21(6):1311-1321

54. Zhou L, Cryan EV, D’Andrea MR, Belkowski S, Conway BR, Demarest KT (2003) Human cardiomyocytes express high level of $\mathrm{Na}+$ /glucose cotransporter 1 (SGLT1). J Cell Biochem 90(2):339-346

55. von Lewinski D, Gasser R, Rainer PP, Huber MS, Wilhelm B, Roessl U et al (2010) Functional effects of glucose transporters in human ventricular myocardium. Eur J Heart Fail 12(2):106-113

56. Sayour AA, Olah A, Ruppert M, Barta BA, Horvath EM, Benke $K$ et al (2020) Characterization of left ventricular myocardial sodium-glucose cotransporter 1 expression in patients with endstage heart failure. Cardiovasc Diabetol 19(1):159

57. Kohan DE, Fioretto P, Tang W, List JF (2014) Long-term study of patients with type 2 diabetes and moderate renal impairment shows that dapagliflozin reduces weight and blood pressure but does not improve glycemic control. Kidney Int 85(4):962-971

58. Strojek K, Yoon KH, Hruba V, Elze M, Langkilde AM, Parikh $S$ (2011) Effect of dapagliflozin in patients with type 2 diabetes who have inadequate glycaemic control with glimepiride: a randomized, 24-week, double-blind, placebo-controlled trial. Diabetes Obes Metab 13(10):928-938

59. Wilding JP, Woo V, Soler NG, Pahor A, Sugg J, Rohwedder K et al (2012) Long-term efficacy of dapagliflozin in patients with type 2 diabetes mellitus receiving high doses of insulin: a randomized trial. Ann Intern Med 156(6):405-415

60. Cefalu WT, Leiter LA, de Bruin TW, Gause-Nilsson I, Sugg J, Parikh SJ (2015) Dapagliflozin's effects on glycemia and cardiovascular risk factors in high-risk patients with type 2 diabetes: a 24-week, multicenter, randomized, double-blind, placebocontrolled study with a 28 -week extension. Diabetes Care 38(7):1218-1227 